\title{
CORTICAL ATROPHY OF THE BRAIN IN INFANTS ASSOCIATED WITH FAILURE TO THRIVE
}

\author{
BY \\ P. M. SMYTHE and I. A. D. BOUCHIER \\ From the Department of Child Health, University of Cape Town and Groote Schuur Hospital
}

(RECEIVED FOR PUBLICATION SEPTEMBER 4, 1959)

Infants may fail to thrive for a variety of reasons. When recognized causes such as congenital abnormalities, infections, defective intake and absorption, metabolic and endocrinal disturbances have been excluded, there still remains a group in which no satisfactory explanation is readily apparent. One cause which may account for some of these marasmic infants, and which does not appear to have been previously recorded, is cortical atrophy of the brain. Attention was drawn to this possibility by finding a hemiplegic infant who would not gain weight. An air encephalogram (A.E.G.) revealed cortical atrophy of the brain. Subsequently A.E.G.s were done on other infants, even if they had no clinical signs of damage to the nervous system. In all of them, unless stated to the contrary, the urine, chest radiograph, Mantoux test, Wassermann reaction, blood urea and serum chemistry were normal. Air encephalograms were only done if, after two months of adequate diet in hospital, there was no satisfactory reason found for the failure of these infants to gain weight.

Lumbar A.E.G.s were done under sedation with seconal and largactil. Spinal fluid was replaced by an equivalent volume of air. The total volume of air varied from case to case, but in no child was an excessive amount injected, nor was there any increase in intracranial pressure as judged by bulging of the fontanelle during or following the procedure. No ill effects following A.E.G. were seen in any of the infants.

\section{Case Histories}

Case 1. V.W., aged 4 months, was admitted on November 28, 1955, weighing $6 \mathrm{lb}$. $7 \mathrm{oz}$. Birth weight was said to be $3 \mathrm{lb} .3 \mathrm{oz}$. The only abnormal physical signs elicited were a mild right spastic hemiparesis and a head circumference of $13 \cdot 25$ in. A radiograph showed two well-developed centres of ossification in the carpal bones. Eleven weeks after admission the child weighed $6 \mathrm{lb} .15 \mathrm{oz}$. A.E.G. showed some ventricular dilatation and excessive cortical air suggesting atrophy. By the age of 2 years 9 months the child had grown fairly well, her head circumference was $17 \cdot 75$ in., but the hemiplegia was marked and she had difficulty in speaking.

Case 2. C.P., aged 6 weeks, was admitted on February 7, 1956, weighing $7 \mathrm{lb}$. $6 \mathrm{oz}$. Labour and pregnancy were normal. The infant sucked poorly, vomited intermittently and failed to thrive. Fifteen weeks after admission she weighed $7 \mathrm{lb} .5 \mathrm{oz}$. A barium swallow and meal revealed nothing abnormal. She was somewhat slow in her development but had no abnormal neurological signs. An A.E.G. at the age of 6 months was only contemplated because of the findings in Case 1. It showed excessive cortical air compatible with atrophy.

Case 3. S.G., aged 7 months, was admitted on March 17, 1956, weighing $9 \mathrm{lb} .6 \mathrm{oz}$. The pregnancy had been normal but the infant had been born by breech delivery. The child sucked poorly. She had a bronchopneumonia and it was noted that she could not sit. She was re-admitted at 10 months, weighing $11 \mathrm{lb} .10 \mathrm{oz}$, because of vomiting and failure to thrive. A barium meal showed a hiatus hernia. Vomiting improved somewhat on medical treatment but at a year she still could not sit unsupported. There were no abnormal neurological signs except occasional spasms of the eyes of an oculogyric type. The head circumference was 16 in. Eleven weeks after admission she weighed 11 lb. 14 oz. An A.E.G. showed gross evidence of cortical atrophy. Follow-up at 3 years 9 months found the child to be mentally very retarded, weighing $20 \mathrm{lb}$. $6 \mathrm{oz}$. and with a head circumference of $18 \mathrm{in}$.

Case 4. J.V., aged 1 year, because of failure to thrive, was admitted on September 18, 1957, from the Skin Ward where she was under treatment for eczema, hepatosplenomegaly and anaemia. She weighed $9 \mathrm{lb}$. $14 \mathrm{oz}$. The head circumference was $14 \cdot 5 \mathrm{in}$. She had nystagmus and could not hold up her head. The serum cholesterol, bone marrow and a radiograph of the long bones were all normal. A.E.G. showed evidence of gross cortical atrophy and ventricular dilatation. The child died weighing $8 \mathrm{lb} .2 \mathrm{oz}$. 
Case 5. A.L. was admitted on September 16, 1957, also from the Skin Wards, where she had been found to be suffering from ectodermal dysplasia. The blood urea varied between 56 and $72 \mathrm{mg} . / 100 \mathrm{ml}$. An intravenous pyelogram showed a normal pattern on the right side and delayed secretion from the left kidney. The child gained $12 \mathrm{oz}$. in weight in 8 weeks, and at 7 months weighed 8 lb. 12 oz. An A.E.G. showed some ventricular dilatation and gross cortical atrophy.

Case 6. S.J., aged 2 weeks, was admitted on June 10, 1957, weighing $5 \mathrm{lb} .11 \mathrm{oz}$. Labour and pregnancy had been normal. There was frequent vomiting and at the age of 12 weeks she weighed $5 \mathrm{lb} .15 \mathrm{oz}$. Subsequent weight gain was slow. An A.E.G. at 5 months showed cortical atrophy as well as some subdural air.

Case 7. V.M., aged 2 weeks, was admitted on December 30, 1957, weighing $3 \mathrm{lb}$. $3 \mathrm{oz}$. She had had a minor convulsive episode on the third day of life. The first 8 months of life were spent in hospital during which time she gained weight slowly. She could then hold up her head but could not sit even with support. Both optic discs were pale but she could see and followed objects without making any effort to grasp. A radiograph showed three carpal centres of ossification. An A.E.G. at 8 months showed slight ventricular dilatation and cortical atrophy. A carotid angiogram was done on the right side and showed a normal anatomy of the cerebral vessels. The child died when 16 months of age, weighing $10 \mathrm{lb}$.

\section{Interpretation of Air Encephalograms}

Smith and Crothers (1950) have emphasized the need to interpret, with reserve, air shadows lying over the brain cortex. The common error is to

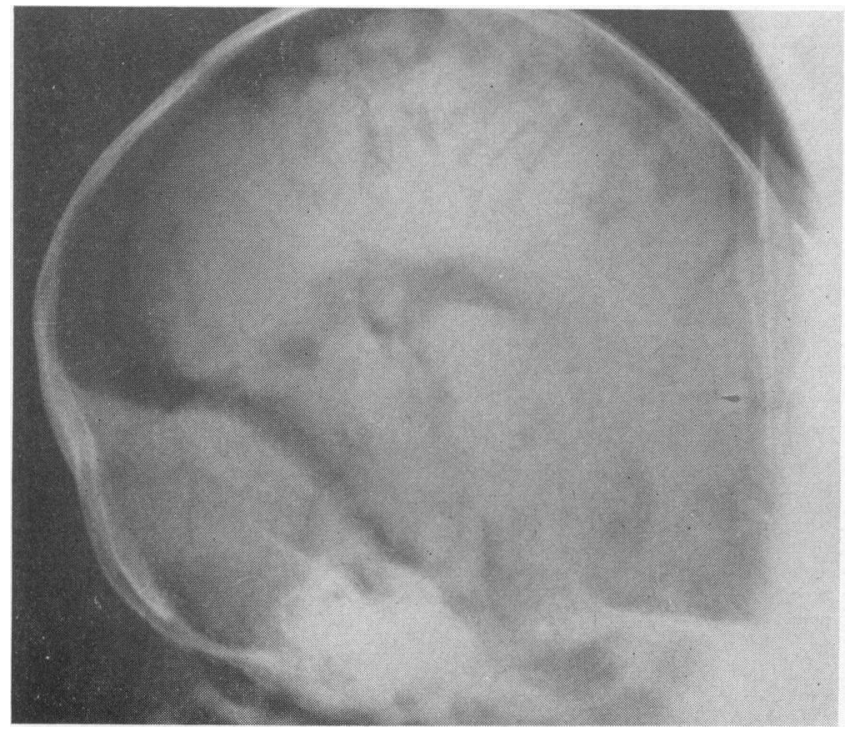

Fig. 1.-Subdural air: showing extension along the tentorium cerebelli. diagnose cortical atrophy because air has entered the subdural space. Subdural air can be recognized by the following features: (1) It moves with alteration of posture of the head. (2) It dips down beside the falx and along the tentorium cerebelli (see Fig. 1). (3) It is smoothly lined and is associated with fluid levels (Fig. 2).

Cortical atrophy can be recognized in the following way: (1) The frontal and parietal regions are predominantly affected (Fig. 3). (2) It produces an irregular picture of peaks and valleys the dilated sulci being usually more than $3 \mathrm{~mm}$. wide (Figs. 4 and 5). (3) If the picture is associated with ventricular dilatation it is diagnostic, but it must be stressed that considerable atrophy of the cortex can occur and yet the ventricles may appear to be normal in size.

Judged by these criteria, all cases in this series had cortical atrophy. It is recognized, however, that a positive diagnosis of brain atrophy based solely on the A.E.G. appearances is not justified. All the infants were at an age when normally rapid brain growth occurs and the changes seen on A.E.G. might have been in part, if not wholly, due to a primary defect in growth rather than an atrophy. No separation of the two types of defect is possible on the evidence available, and in this paper the term atrophy may in fact be covering both a primary defect in cerebral growth and atrophy due to brain damage.

Three facts seem to confirm the diagnosis of cortical atrophy made from these A.E.G.s. Firstly, in those children who have survived and have been followed up, if it was not obvious at first, there has been subsequent clear evidence of brain damage. Secondly, the A.E.G.s of those children with clinical evidence of brain damage differed in no way from those who showed no clinical evidence of damage to the nervous system at the time of A.E.G. Thirdly, confirmation of cortical atrophy was found at necropsy in two other children who had similar A.E.G. changes, but who are not included in this series as their physical development was normal.

It must be stressed that the presenting symptom in these infants was failure to thrive. In Cases 2, 6 and 7 the possibility of cortical brain damage was not at first suspected. These infants were still very young and it was thought that any slowness of intellectual development could be accounted for 


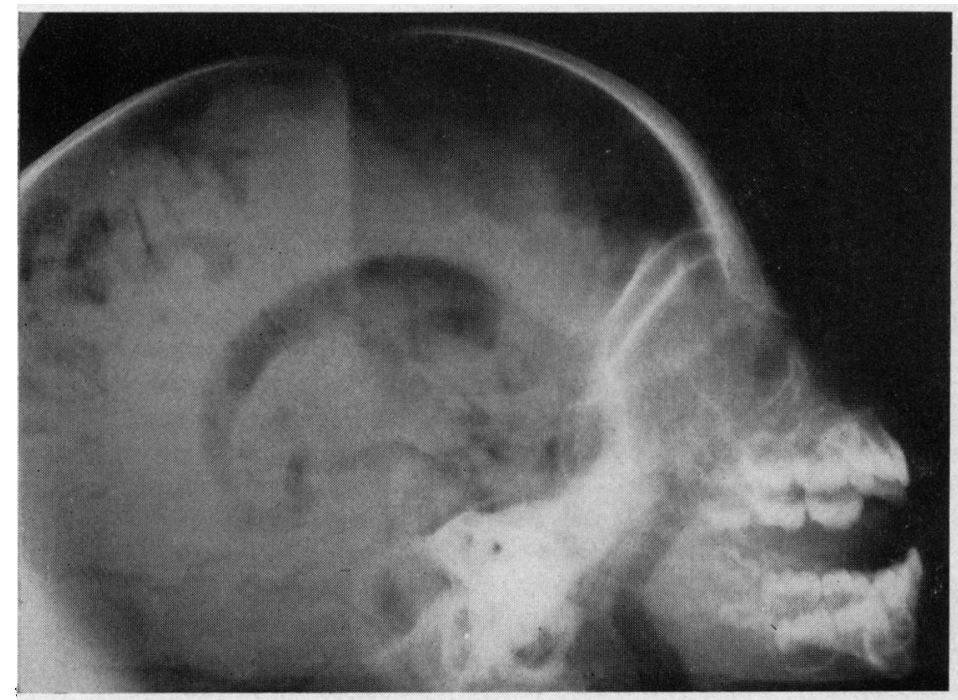

FIG. 2.

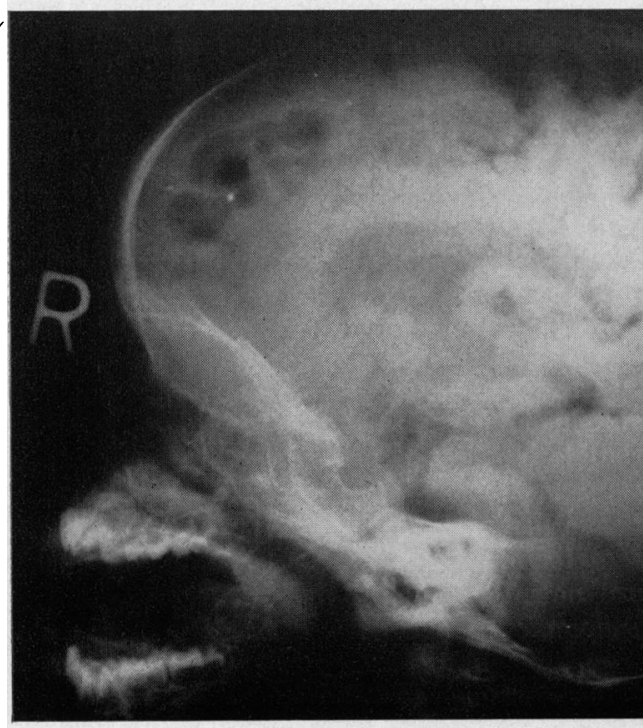

FIG. 3.

FIg. 2.-Subdural air: showing a fluid level.

FIG. 3--Cortical atrophy: showing predominantly parietal involvement.

FIG. 4.-Cortical atrophy: A.P. view showing widening of the sulci.

FIG. 5.-Cortical atrophy: lateral view showing widening of sulci.
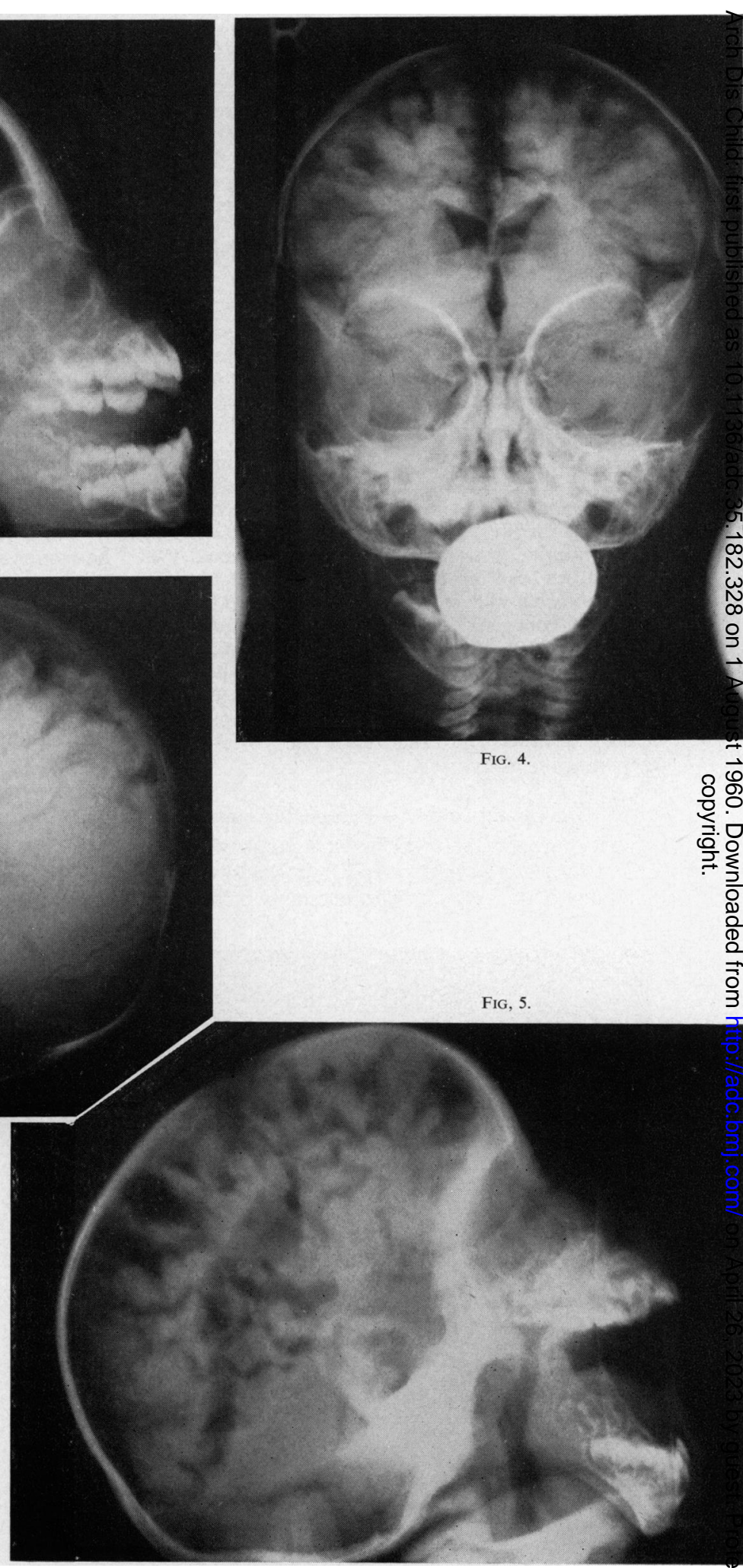

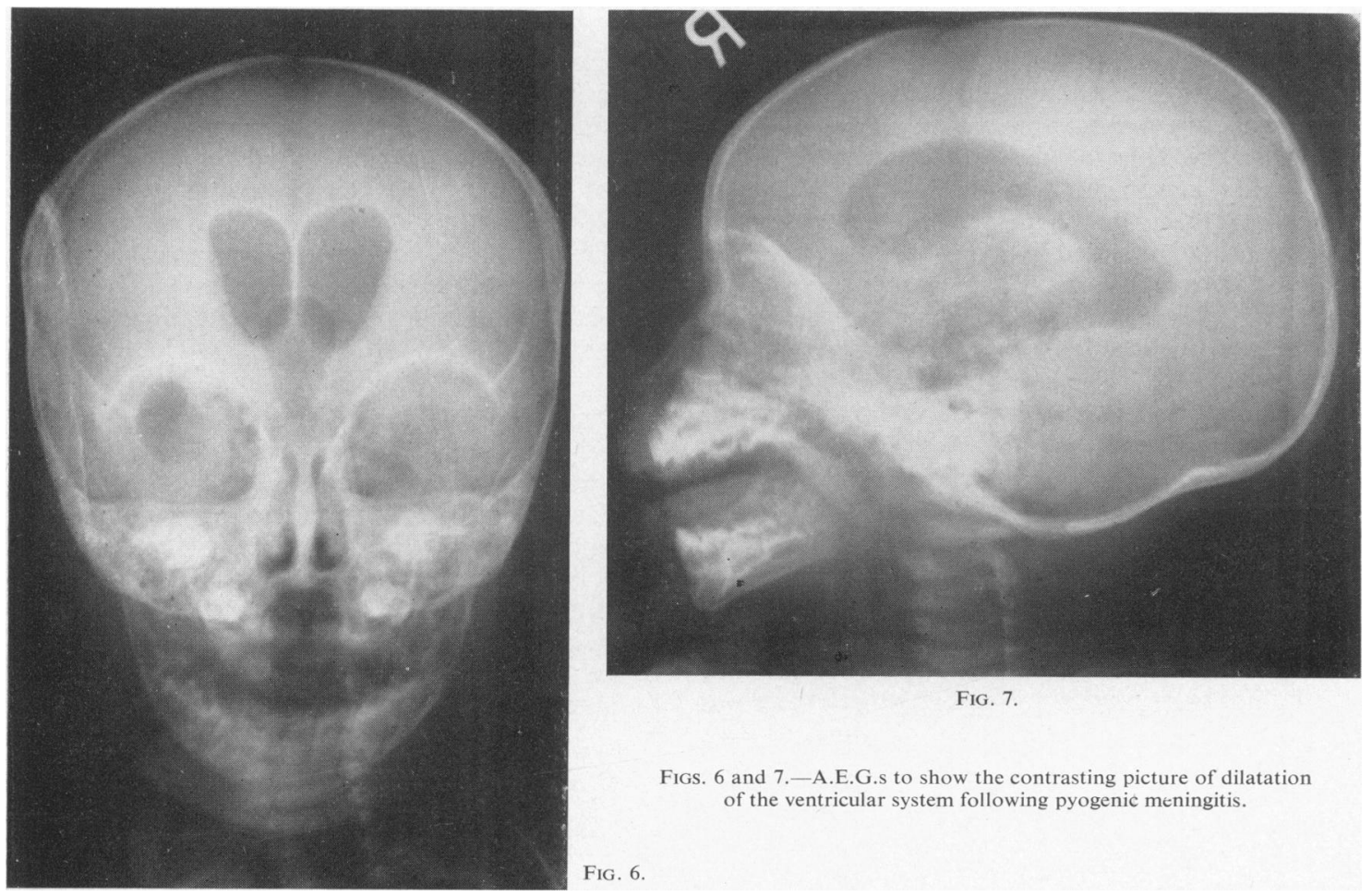

FIG. 7.

Figs. 6 and 7.-A.E.G.s to show the contrasting picture of dilatation of the ventricular system following pyogenic meningitis.

by the failure of physical growth. Only later, after the infants had been followed for scme months, was it realized that the cerebral damage was probably the basic abnormality. Cases 1 and 3 differed from the others only in that they had neurological signs which drew attention to the brain damage. Involvement of areas of the brain that produce definite abnormal physical signs does not, however, seem to be an adequate reason for separating this group from those with no neurological signs. In Cases 4 and 5 there was associated disease that probably contributed to, if it was not the major cause of, the failure to gain weight. Their inclusion seems justified as they showed a similar clinical state and the same changes on their A.E.G.s.

One can only speculate on the cause of the atrophy. There was no familial incidence to suggest a genetic factor. In only one infant was an arteriogram done and this showed no evidence of a vascular defect. It is most unlikely that the cause was a meningitis. Some history of a previous illness should have been obtainable. Furthermore, A.E.G.s done on infants who have had meningitis show an entirely different picture with dilatation of the ventricular system, very little if any air over the cortex and rarely any subdural air (Figs. 6 and 7).
In fact the readiness with which the ventricles of children, who have had meningitis, fill, suggests that some other factor plays a part, and that this is possibly dilatation of the whole of the ventricular system including the aqueduct which allows a freer efflux of ventricular fluid and its replacement with air. The atrophy is most likely to be the result of cerebral anoxia, probably intra-uterine or neonatal. Frontal and parietal involvement occurs frequently in such circumstances, and the small size of the heads of these infants also supports the concept of the damage occurring at an early age.

There are many factors governing normal growth and in general growth may be impaired for three main reasons: (1) when diet fails to supply the required nutrients; (2) when there is some breakdown in the physiological process of transporting the nutrients to the tissues; and (3) when the body fails to utilize the nutrients.

Keys (1953) believes that the only neurological causes of failure to thrive are those diseases causing loss of appetite, and those neurological lesions which interfere with feeding. At present no neurological 'centres for growth' are known. Brobeck (1946) states that the hypothalamus normally participates in the maintenance of the overall energy equilibrium 
and Brock (1953) ascribes this function to the thalamic hypothalamic level. Lesions of the pituitary might be expected to show delayed ossification of the centres of bone growth. In those children of this series where radiographs of the carpus had been taken, the bone age appeared normal. After damage to the frontal lobes, both clinically and experimentally, a weight loss is frequently observed. Because these subjects frequently manifest voracious appetites, this loss of weight has been ascribed to hypermobility of the intestinal tract (Fulton, Jacobsen and Kennard, 1932). The converse also applies, and a great diminution in appetite, depending on altered prefrontal cortical function, has been described (Brock, 1953). Thus the prime effect of neuronal damage has been an alteration in the hunger drive, with only a secondary nutritional effect. In three of the infants of this series, vomiting apparently of a cerebral type, must have affected growth although it did not always appear either severe or frequent enough to be an entirely satisfactory explanation for the failure to gain weight. In two others there was no vomiting, feeding was normal and appetites were reasonable. When there was some impairment of appetite, tube feeding still failed to achieve a reasonable weight gain. This would suggest that, in some children, cortical atrophy through some subtle mechanism has an adverse effect on the general metabolism resulting in a failure to thrive. The fact that other children with clinical and A.E.G. evidence of cortical atrophy had normal physical development raises the possibility of there being some 'centre for growth' which is affected in some cases and not in others.
In conclusion, it is recognized that the diagnosis of cortical atrophy of the brain is easily made as these infants grow older; but it would be helpful to know the cause of failure to thrive in very young infants when the recognition of mental retardation is not so easy; parents and doctors can then be spared a period of uncertainty as to the nature of the child's illness. In addition unnecessarily prolonged hospitalization can be avoided in those hospitals where there is still a tremendous pressure on beds.

\section{Summary}

A series of infants is described in which failure to thrive was the presenting symptom. All of them had heads that were small in circumference and, if not apparent when first seen, subsequently showed evidence of marked mental retardation. All of them had abnormal air encephalograms which were interpreted as indicating cortical atrophy of the brain. In infants the association of cortical atrophy of the brain with failure to thrive does not appear to have been previously recorded.

We wish to thank Professor F. J. Ford for help with the paper; Dr. J. A. Burger, Medical Superintendent of Groote Schuur Hospital, for permission to publish and Mr. Todd for the photographic reproductions.

\section{REFERENCES}

Brock, S. (1953). The Basis of Clinical Neurology. 3rd ed., p. 421. Williams and Wilkins, Baltimore.

Brobeck.J. R. (1946). Mechanism of the development of obesity in animals with hypothalamic lesions. Physiol. Rev., 26, 541 .

Fulton, J. F., Jacobsen, C. F. and Kennard, M. A. (1932). A note concerning the relation of the frontal lobes to posture and forced grasping in monkeys: Brain, 55, 524.

Keys, A. (1952). In Diseases of Metabolism. 3rd ed., pp 573-8. Saunders, Philadelphia.

Smith, H. V. and Crothers, B. (1950). Subdural fluid as a consequence of pneumoencephalography. Pediatrics, 5, 375. 